Ophthalmologe 2010 $107: 402$

DOI 10.1007/s00347-009-2061-7

๑) Springer-Verlag 2010

G. Geerling

Klinik für Augenheilkunde, Würzburg

\title{
Management komplexer Glaukome - Henne oder Ei?
}

steroidinduzierten Erhöhung des intraokularen Drucks und einer damit verbundenen bleibenden Sehminderung gelehrt und beachtet werden.

In einer Zeit, in der fachliche Allrounder (engl: „comprehensive ophthalmologist") zu einer bedrohten Spezies werden, sollte subspezialisiertes Wissen in einem ganzheitlichen Behandlungskonzept zusammengeführt werden. Beispielhaft hierfür sei die Subspezialität des Kinderophthalmologen genannt, die es in dieser Form im angloamerikanischen Raum, aber kaum in Deutschland gibt. Bei der Betrachtung der Kombinationen von Glaukom und Hornhaut, Katarakt, Netzhaut oder Uveitis wird schnell klar, dass sich oft die Frage nach der Ursache-Wirkungs-Beziehung im Sinne eines Henne-oder-Ei-Problems nicht beantworten lässt und dennoch die komplexe Situation subspezialitätenübergreifend behandelt werden muss. Gefahr von relativen Skotomen für übrige Belange. Bei allem Respekt vor der angesichts der Expansion unseres pathophysiologischen Verständnisses, diagnostischen Wissens und therapeutischen Möglichkeiten - erforderlichen Beschränkung auf kleinere Ausschnitte der Augenheilkunde bleiben das Auge und die okuläre Adnexe eine anatomische und funktionelle Einheit. Nicht zuletzt aufgrund der räumlichen Begrenzung des Organs Auge sind die Subspezialitäten auf eine enge Kooperation angewiesen und müssen sich einen offenen Blick für benachbarte Probleme erhalten. So muss beispielhaft bereits in den Ausbildungsjahren auch und gerade in einer Hornhautsprechstunde die Gefahr einer
Der Begriff Komplex stammt vom lateinischen completari (umarmen, umfassen). Komplexität ist das Gegenteil von Einfachheit und beschreibt eine Situation, deren Behandlung aufgrund von zu vielen und in der Summe zu komplizierten Details erschwert ist. Glaukomerkrankungen können eine solche komplexe Situation darstellen. Das Leitthema dieser Ausgabe von „Der Ophthalmolog“ stellt das Management dieser Komplexi-

\section{( Subspezialisiertes Wissen sollte in einem ganzheitlichen Behandlungskonzept zusammengeführt werden}

tät aus der Sicht verschiedener Subspezialitäten dar. Wir hoffen, dass uns dies nicht nur umfassend, sondern für den Leser auch praxisrelevant gelungen ist.

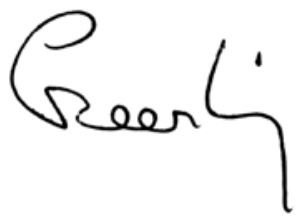

Gerd Geerling

\section{Korrespondenzadresse \\ Prof. Dr. G. Geerling \\ Klinik für Augenheilkunde \\ Joseph-Schneider-Straße 11, 97080 Würzburg \\ g.geerling@klinik.uni-wuerzburg.de}

